\title{
Fibroblasts from phenotypically normal palmar fascia exhibit molecular profiles highly similar to fibroblasts from active disease in Dupuytren's Contracture
}

\author{
Latha Satish $^{1 *}$, William A LaFramboise ${ }^{2}$, Sandra Johnson ${ }^{3}$, Linda Vi ${ }^{4}$, Anna Njarlangattil ${ }^{4}$, Christina Raykha ${ }^{4}$, \\ John Michael Krill-Burger², Phillip H Gallo, David B O'Gorman ${ }^{4}$, Bing Siang Gan ${ }^{4}$, Mark E Baratz ${ }^{5}$, Garth D Ehrlich ${ }^{3}$ \\ and Sandeep Kathju ${ }^{1}$
}

\begin{abstract}
Background: Dupuytren's contracture (DC) is a fibroproliferative disorder characterized by the progressive development of a scar-like collagen-rich cord that affects the palmar fascia of the hand and leads to digital flexion contractures. DC is most commonly treated by surgical resection of the diseased tissue, but has a high reported recurrence rate ranging from $27 \%$ to $80 \%$. We sought to determine if the transcriptomic profiles of fibroblasts derived from DC-affected palmar fascia, adjacent phenotypically normal palmar fascia, and non-DC palmar fascial tissues might provide mechanistic clues to understanding the puzzle of disease predisposition and recurrence in DC.

Methods: To achieve this, total RNA was obtained from fibroblasts derived from primary DC-affected palmar fascia, patient-matched unaffected palmar fascia, and palmar fascia from non-DC patients undergoing carpal tunnel release (6 patients in each group). These cells were grown on a type-1 collagen substrate (to better mimic their in vivo environments). Microarray analyses were subsequently performed using Illumina BeadChip arrays to compare the transcriptomic profiles of these three cell populations. Data were analyzed using Significance Analysis of Microarrays (SAM v3.02), hierarchical clustering, concordance mapping and Venn diagram.

Results: We found that the transcriptomic profiles of DC-disease fibroblasts and fibroblasts from unaffected fascia of DC patients exhibited a much greater overlap than fibroblasts derived from the palmar fascia of patients undergoing carpal tunnel release. Quantitative real time RT-PCR confirmed the differential expression of select genes validating the microarray data analyses. These data are consistent with the hypothesis that predisposition and recurrence in DC may stem, at least in part, from intrinsic similarities in the basal gene expression of diseased and phenotypically unaffected palmar fascia fibroblasts. These data also demonstrate that a collagen-rich environment differentially alters gene expression in these cells. In addition, Ingenuity pathway analysis of the specific biological pathways that differentiate DC-derived cells from carpal tunnel-derived cells has identified the potential involvement of microRNAs in this fibroproliferative disorder.

Conclusions: These data show that the transcriptomic profiles of DC-disease fibroblasts and fibroblasts from unaffected palmar fascia in DC patients are highly similar, and differ significantly from the transcriptomic profiles of fibroblasts from the palmar fascia of patients undergoing carpal tunnel release.
\end{abstract}

\footnotetext{
* Correspondence: latsat@hotmail.com

'Department of Surgery, Division of Plastic Surgery, University of Pittsburgh

Medical Center, Pittsburgh, PA, USA

Full list of author information is available at the end of the article
} 


\section{Background}

Dupuytren's contracture (DC) is characterized by abnormal thickening of palmar fascia into collagen-rich cords that cause the fingers to bend and curl into a flexed and contracted state [1]. Although this disease can occur in both sexes, it is more common in men of Northern European descent [2-4] and typically presents in the 4th to 6th decade of life. DC has been reported to behave as a heritable genetic disorder, with evidence that it arises (in at least some cases) from an autosomal dominant gene on chromosome 16 with variable penetrance [3]. Lifestyle factors including smoking or heavy drinking $[5,6]$, and heavy manual labor and hand trauma, have also been linked to development of DC $[7,8]$, as have diabetes, epilepsy and hypercholesterolemia [9-11].

Treatment of DC remains problematic. A variety of non-surgical interventions, including injection of steroids [12] or gamma-interferon [13], use of creams based on vitamin E [14], dimethyl sulphoxides [15], and ultrasound therapy [16] etc. yield limited benefits. Recently, direct injection of clostridial collagenase has been evaluated with some promising results [17-19]. However, surgical excision of the involved contracted tissue remains the mainstay of therapy, supplemented with post-operative splinting and physical therapy [20,21]. Alternative therapeutic approaches remain desirable since surgery carries significant risks, including damage to the digital nerves and blood vessels, damage to the underlying flexor tendons, and wound healing failure with the possibility of skin necrosis.

A particularly vexing feature of DC is its propensity for recurrence despite the appearance of successful initial treatment. Many patients eventually require multiple surgeries with a cumulative risk of morbidity. However, it remains unclear what factors are responsible for recurrence of the disease. Apart from the possibility of a genetic predisposition, it has been hypothesized that undetected residual foci of incipient disease are present in the otherwise normal appearing and uninvolved palmar fascia, and that these cells represent sites of disease recurrence.

We have previously investigated the transcriptomic differences between fibroblasts derived from diseased DC cords versus fibroblasts from phenotypically normal palmar fascia in patients undergoing carpal tunnel (CT) release. These studies demonstrated intrinsic differences in gene expression between these cell populations that persisted even after propagation under cell culture. We have now extended these studies to include fibroblasts from macroscopically uninvolved (i.e. phenotypically normal) palmar fascia surgically removed from patients with DC. These fibroblasts, as well as fibroblasts from diseased DC cords and control carpal tunnel fibroblasts, were cultivated in cell culture on a type- 1 collagen substrate to better approximate the in vivo collagen-enriched environment that these cells experience. The transcriptomic signatures of these three cell types were then compared to answer the question: do fibroblasts from phenotypically normal palmar fascia in DC more closely resemble their counterparts in phenotypically dissimilar DC cords, or cells from phenotypically similar carpal tunnel fascia?

\section{Methods \\ Clinical specimens}

Dupuytren's contracture (DC) cord samples and small samples of phenotypically normal palmar fascia tissue (PF) were surgically explanted at the Hand and Upper Limb Centre at St Joseph's Health Care (SJHC), London, ON, Canada. We also obtained phenotypically normal palmar fascia from patients undergoing carpal tunnel release (CT) in London, ON, Canada, and at the Allegheny General Hospital, Pittsburgh, PA. The study protocol conformed to the ethical guidelines of the 1975 Declaration of Helsinki. All specimens were collected under Institutional Review Board approval. Six patient samples of each tissue type were used to derive fibroblasts for use in these studies.

\section{Primary cell culture}

Primary cultures of fibroblasts were purified from the surgically resected DC cord and matching specimens of normal appearing palmar fascia (PF), and from specimens of normal palmar fascia of patients undergoing carpal tunnel surgery (CT) as previously described [22,23]. The cultures were maintained in $\alpha$-MEM-medium supplemented with $10 \%$ fetal bovine serum (FBS, Invitrogen Corporation, Carlsbad, CA) and 1\% antibiotic-antimycotic solution (Sigma-Aldrich, St Louis, MO). All cultures were harvested prior to the sixth passage, with no changes in cell morphology observed during the in vitro expansion protocol. For the present study we used 6 primary DC and patient-matched PF cell cultures as well as primary fibroblasts purified and expanded from 6 patients undergoing surgical carpal tunnel release. Primary cultures of fibroblasts were grown on collagen monolayers as described previously [24]. In brief, collagen was coated on 6-well tissue culture plates with each well containing $880 \mu \mathrm{l}$ of rat tail (type-1) collagen and $200 \mu \mathrm{l}$ of the neutralization solution (2 parts $0.34 \mathrm{~N} \mathrm{NaOH}$ and 3 parts $10 \mathrm{x}$ Waymouth media) to a final concentration of $1.9 \mathrm{mg} / \mathrm{ml}$. Following collagen polymerization, primary cultures were grown in $\alpha$-MEM-medium containing 10\% FBS and $1 \%$ antibioticantimycotic solution and the cultures were left undisturbed at $37^{\circ} \mathrm{C}$ at $5 \% \mathrm{CO}_{2}$ for 72 hours.

\section{Total RNA extraction}

Each well containing cells grown on collagen was treated with $1 \mathrm{ml}$ of $0.25 \mathrm{mg} / \mathrm{ml}$ of collagenase XI (Sigma-Aldrich) at $37^{\circ} \mathrm{C}$ with gentle rotation for 20 mins, until the cells 
detached from the underlying collagen substrate. The samples were collected and centrifuged for 4 minutes at $900 \mathrm{rpm}$ to concentrate the cells into a pellet for RNA purification. Total RNA was extracted using the RNeasy Mini Kit (Qiagen Inc., Valencia, CA) according to the manufacturer's instructions. The concentration of the extracted RNA was quantified using a Nanodrop ND-1000 Spectrophotometer (NanoDrop, Wilmington, DE) (absorption ratio $260 / 280>1.8)$. The samples were then analyzed on an Agilent 2100 Bioanalyzer (Agilent Technologies, Santa Clara, CA) to ensure adequate sample quality and absence of RNA degradation (RIN value > 8.0).

\section{Microarray assays}

Total RNA (1.5 $\mu \mathrm{g} / \mathrm{sample})$ purified from fibroblasts derived from DC cord $(n=6), P F(n=6)$ and CT $(n=6)$ specimens was subjected to analysis with Human WG-6 v3.0 Expression BeadChips (Illumina, SanDiego, CA), each of which contains six arrays on a single BeadChip. Each array is comprised of $>48,000$ probes derived from human genes in the NCBI RefSeq and UniGene databases. Each array on the BeadChip thus provides genome-wide transcriptional representation of well-characterized genes, gene candidates, and splice variants. A total of 18 separate samples were interrogated on the BeadChip arrays, i.e., there was no pooling of samples within groups. This allowed us to evaluate sample to sample variability in gene expression both within and across each group.

Total RNA was amplified using the Illumina ${ }^{\circledR}$ TotalPrep ${ }^{\mathrm{TM}}$ RNA Amplification Kit (Ambion, Austin, TX). Labeling and hybridization was performed according to the Illumina gene expression protocol, featuring a reverse transcription step to synthesize first strand cDNA followed by addition of second strand master mix containing DNA polymerase for second strand cDNA synthesis. This was followed by a single in vitro transcription (IVT) amplification step that incorporated biotin-labeled nucleotides. Subsequent steps included array hybridization, washing, blocking, and streptavidin-Cy3 staining followed by serial non-stringent washing steps to remove unbound conjugate. Following the final rinse, the bioarrays were dried by centrifugation and scanned. The Illumina bioarrays were read in an Illumina BeadArray Reader and the primary intensity data were obtained in standard file format, using Genome Studio software.

\section{Microarray expression analysis}

The data obtained from the three cell sources were analyzed for differences in expression as previously described using the multiclass analysis tool of the Significance Analysis of Microarrays program (SAM version 3.02) [23,25]. We then performed unsupervised hierarchical clustering, concordance mapping and created Venn diagrams using the Partek Genomics Suite version 6.4 (St. Louis, MO) to evaluate similarities and differences in the gene expression patterns among the fibroblasts from each of the three cell sources.

\section{Pathway analysis}

Statistically significant and differentially expressed genes determined by SAM were subjected to systematic network analysis to determine the primary biological processes and pathways associated with each group using "Ingenuity Pathways Analysis" (IPA, ver.5.0, Ingenuity ${ }^{\circledR}$ Systems, http://www.ingenuity.com, Mountain View, CA). Only genes that were significantly increased or decreased were included as a defined parameter for the core analysis. Using information stored in the Ingenuity Pathways Knowledge Base (IPKB), genes that mapped to genetic networks were ranked by a score based on the number of eligible molecules contained in the network, which also defines the probability that each network can be achieved by chance alone. A score of 3 is considered threshold of significance which means that there is a 1 in 1000 chance that genes are integrated in the network by pure chance. Canonical pathways were also identified from the IPA library. The significance of the association between the data set and the canonical pathway was measured in two ways within the Ingenuity program: i) as a ratio of the number of genes from the data set that map to the pathway divided by the total number of genes that map to the canonical pathway was calculated, and ii) by the Fischer's exact test which was used to calculate a $p$-value determining the probability that the association between dataset genes and the canonical pathway is significant.

\section{Quantitative real time RT-PCR}

The protocol for reverse transcription reactions and real time PCR was followed as previously described [23]. Ninety ng of total RNA and $150 \mathrm{ng}$ of random primers (Invitrogen Corporation) were used in $30 \mu \mathrm{L}$ reactions and real time RT-PCR was performed with triplicate sample reads. The 18 samples were prepared separately such that gene expression analysis was performed using individual samples without pooling. Real time PCR amplification and detection of template was carried out using Applied Biosystems transcript-specific assays including: angiopoietin-like 7 (ANGPTL7) - Hs00221727_m1, Laminin alpha 5(LAMA5) - Hs00966585_m1, Shroom 2 - Hs01113 636_m1, and GAPDH - Hs02758991_g1. Using the comparative critical cycle $(\mathrm{Ct})$ method and using GAPDH as the endogenous control, the expression levels of the target genes were normalized using a 95\% confidence interval. The relative expression of the 18 samples ( 6 for each subset) was averaged and statistical analysis for significance was performed using a Student's $t$-test. Results shown are from two independent experiments performed in triplicate. 


\section{Results}

The raw intensity values representing expression of individual transcripts were corrected by subtraction of the background intensity for each array. All values that were at or below background concomitantly in all 3 sample groups were removed yielding 27214 transcripts for further analysis. Box plot analysis of the distribution of these intensity values across the three groups demonstrated comparable dispersion of the individual data sets regardless of patient source, eliminating the need for log normalization or secondary smoothing of the raw intensity data prior to statistical evaluation (Figure 1).

Transcript intensity values were analyzed for differences in individual gene expression levels using the multiclass comparison of the SAM program (Tusher et al., 2001). The overall raw data distribution was not modified prior to analysis except for median centering of the array data within the SAM program. Results were considered significant at a false detection rate (FDR) of $5 \%(\mathrm{q} \leq 0.05)$ and the multiclass comparison revealed 959 transcripts differentially expressed among the 3 classes (delta $=0.47$ and FDR $=4.89 \%$ ). Removal of cDNA clones, open reading frame transcripts, redundant probes and transcripts for hypothetical proteins among the 959 significant transcripts revealed 724 distinct, functionally annotated gene products that were differentially expressed among the 3 groups (Additional file 1).

Post hoc comparisons were performed on these 959 transcripts against the Student's t-distribution using the Partek Software for paired (Dupuytren samples: DC and PF) and the unpaired samples obtained during carpal tunnel release (CT). Three separate univariate comparisons resulted in 894 transcripts detected as significantly different in DC vs CT-derived fibroblasts, 816 significantly different transcripts between PF and CT-derived fibroblasts, and 308 differentially expressed transcripts between DC and PFderived fibroblasts. These lists are depicted in a Venn Diagram (Figure 2) that illustrates the significant differences specific to each set of individual comparisons (within a circle) versus those shared in more than one of the statistical comparisons (shared between circles). For example, the comparison of DC vs CT-derived fibroblasts yielded 21 transcripts in the non-overlapping area (blue) that were different between these cell types but were not significantly different in the other two comparisons. In contrast, the overlapping region between the PF versus DC circle (green) and the DC vs CT circle (blue) contained 120 transcripts. These 120 transcripts were significantly different in comparisons of DC to either PF or CT-derived fibroblasts but were not different between PF and CT-derived fibroblasts

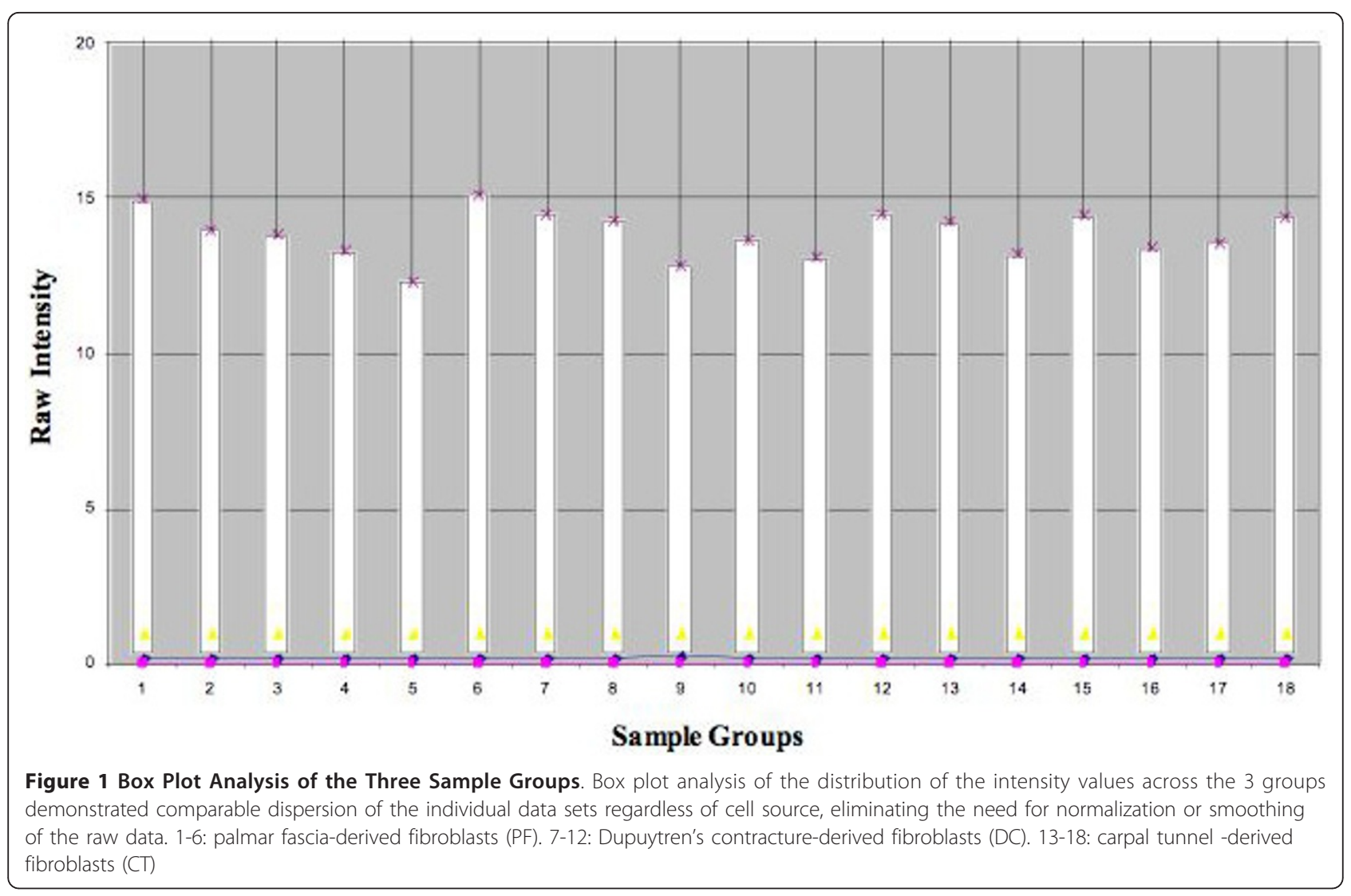




\section{Palmar Fascia vs. Dupuytren}

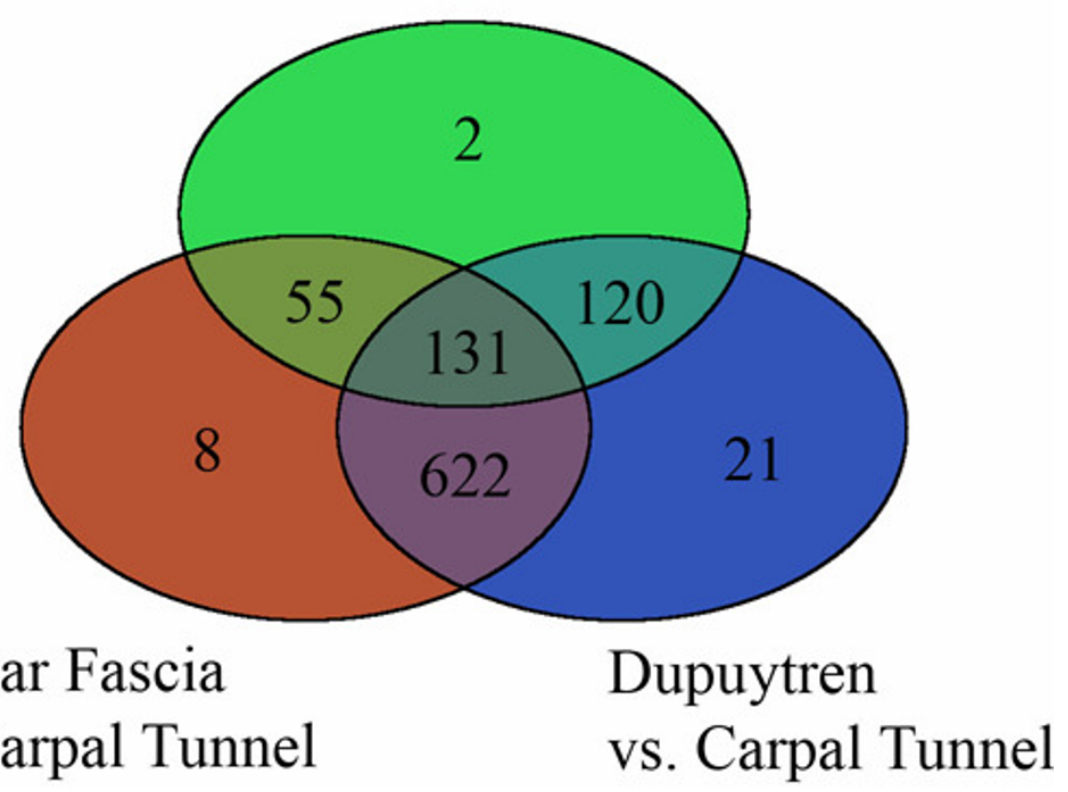

Figure 2 Venn Diagram of Individual Group Comparisons Among the Three Sample Groups. Each of the circles depicts the number of different transcripts based on a binary comparison for each of the labeled sample groups (green, orange, blue) from among the 959 transcripts identified as statistically significant in the study (SAM). Overlapping differences shared among more than one sample group comparison are represented in the areas of intersection between 2 circles. The 131 transcripts in the center of the Venn Diagram represents genes that are different among all group comparisons. The number of transcripts in the carpal tunnel comparisons (622) far exceeded those in the palmar fascia (55) and Dupuytren (120) comparisons

(orange). There were 131 transcripts in the center of the Venn Diagram that overlapped among all three circles, indicating that these transcripts were significantly different in all three comparisons. The most interesting observation, however, is that 622 transcripts (pink) were significantly different in comparisons of CT- to both PF- and DC-derived fibroblasts, but were similarly expressed in DC and PF cells.

Unsupervised hierarchical clustering was performed using the Partek program on log base 2 intensity values of the differentially expressed transcripts identified by the SAM program. Agglomerative clustering was performed to detect dissimilarity based on Euclidean distance with clusters linked based on average values in order to determine if major variations existed in gene expression profiles among the 3 groups [26]. The program was set to delineate the maximum number of clusters based on individual sample comparisons. The primary branch of the resulting dendrogram separated the transcripts of the PF and DC-derived fibroblasts from those of the CTderived fibroblasts, indicating that significant differences in expression were based on patient origin (Figure 3). However, the DC- and PF-derived fibroblasts also formed clearly distinct clusters separate from each other at the next level of branching. Thus, all three cell groups exhibited distinctly different expression signatures with the greatest difference between the unpaired and the paired (DC-derived) samples.

Concordance analysis was performed to delineate similarities in expression profiles among samples. Concordance was calculated using the initial dataset of 959 significant transcripts (log base 2) with similarity between samples based on Euclidean distance (Figure 4). Relative similarity is depicted by a color gradient where blue represents the highest similarity diminishing to the most different values represented in red color. The light blue color at the blue-red interface was indicative of sample identity. Based on this analysis, expression of PF- [1-6] and DC-derived fibroblasts [7-12] exhibited the highest similarity (blue) while both were markedly different from CT-derived fibroblast [13-18] profiles, consistent with the results obtained via hierarchical clustering.

\section{Identification of biological pathways}

Biological interactions among genes differentially expressed between Dupuytren's-derived and CT-derived fibroblasts were identified using Ingenuity ${ }^{\circledR}$ System Analysis. Two distinct gene sets of interest were analyzed: 1) genes concordantly expressed between PF- and CT-derived fibroblasts but significantly different in DC-fibroblasts (120 genes); and 2) genes concordantly expressed between DC- and 


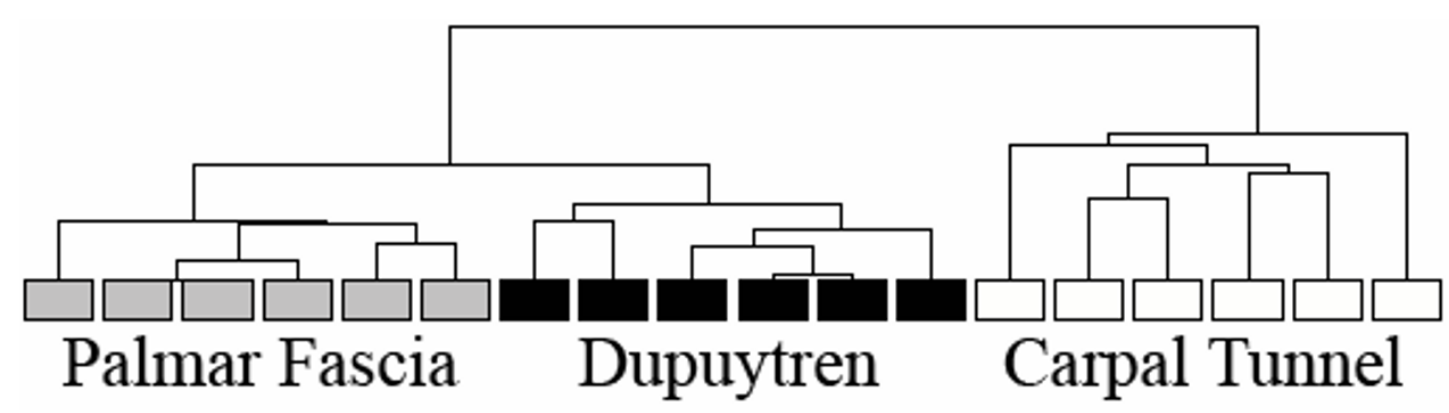

Figure 3 Unsupervised Hierarchical Clustering of Expression Values Based on Sample Origin. The sample distributions of significantly different transcripts (959 transcripts, SAM v. 3.02) are displayed based on unsupervised, hierarchical, agglomerative clustering (Partek: v. 6.4) of log base 2 intensity values. A primary branch of the dendrogram distinguishes the expression values obtained from transcripts of the palmar fascia- and Dupuytren's-derived fibroblasts from those of the carpal tunnel-derived fibroblasts, indicating a distinct difference based on these tissues of origin. Dupuytren's and palmar fascia-derived fibroblasts also formed distinct clusters separate from each other at the next branching level despite their matched patient origin
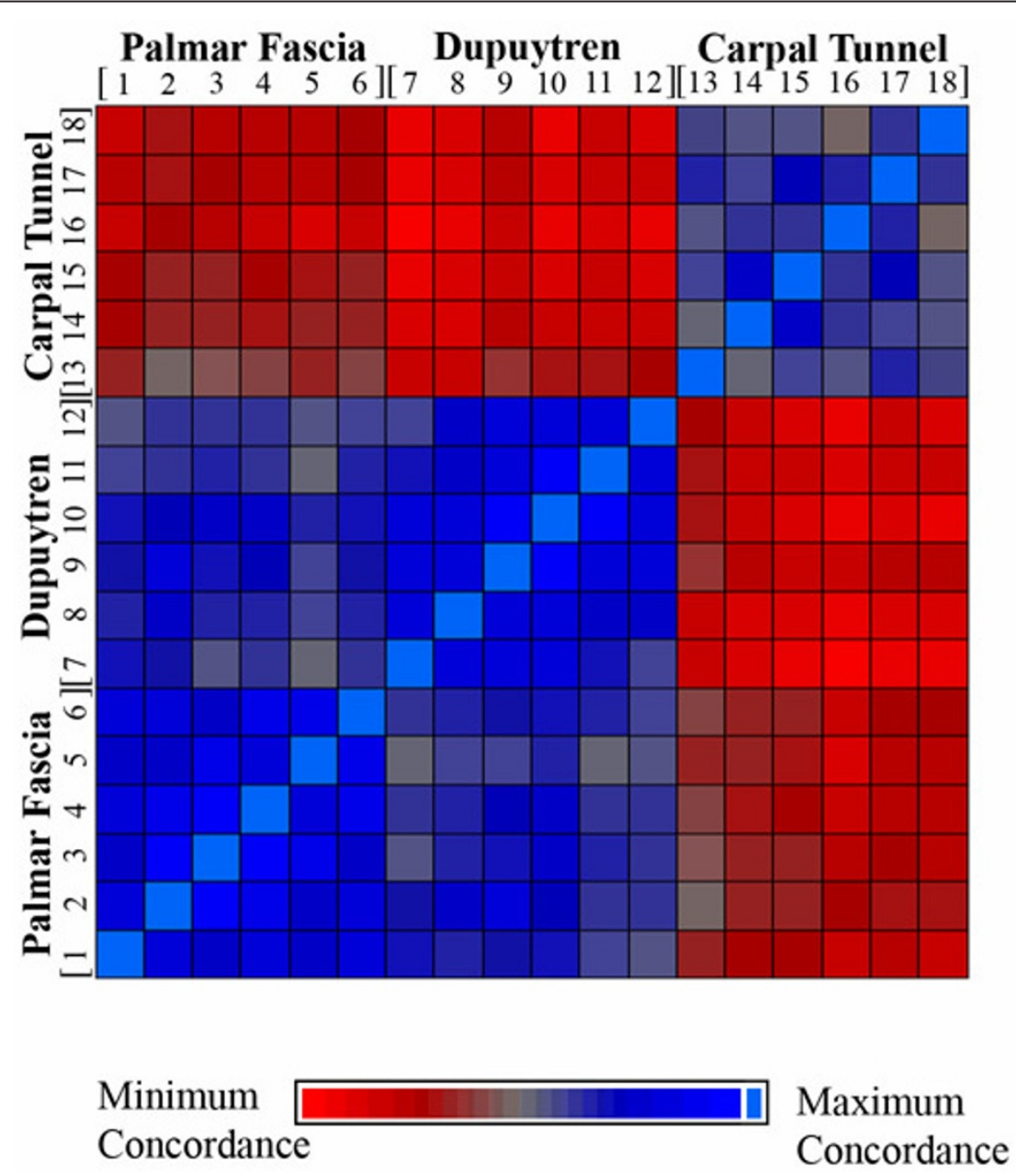

Figure 4 Concordance Analysis of Individual Sample Profiles of Gene Expression. Concordance was calculated (Partek) using the initial dataset of 959 significant transcripts (log base 2) with similarity between samples derived through Euclidean distance based on Euclidian distance. Relative similarity among samples based on labeled origin is depicted through a color gradient where dark blue represents highest similarity of expression values on the scale diminishing across a gradient to the most different values represented in dark red color. The light blue color at the blue-red interface is indicative of sample identity. Based on this analysis, palmar fascia- [1-6] versus Dupuytren-derived fibroblast samples [7-12] exhibited highest similarity (blue), while both were substantially different from carpal tunnel-derived fibroblast [13-18] expression profiles 
PF-fibroblasts but significantly different in CT (622 genes). The program identified 19 and 172 genes respectively that were eligible for function/pathway analysis, comprising 2 and 12 networks respectively with scores of 3 or greater (Tables 1 and 2). The 19 eligible genes identified from the first gene set represented functional categories such as cell death, cell cycle, cellular growth and proliferation- all understandably relevant to a fibroproliferative process like Dupuytren's (Table 1).

In contrast, the 172 genes and 12 networks identified from the second gene set included a much broader array of functional categories, ranging from DNA replication, recombination and repair, to cellular development and cell morphology, to cell-to-cell signaling and interaction. The genes identified were also implicated in a host of other functions including drug metabolism, endocrine system development and function, tumor morphology, molecular transport and even lipid metabolism (Table 2). What role each of these may play in the progression or recurrence of Dupuytren's pathology is as yet unclear.

An interesting feature of the Ingenuity analysis is that the data suggest the involvement of microRNAs (miRNAs) in the dysregulated processes leading to DC pathology. In examining the 120 genes concordantly expressed between PF- and CT-derived fibroblasts but significantly different in DC-fibroblasts, Ingenuity analysis identified two networks which showed direct interaction of three miRNAs, namely miRNA106A, miRNA17 and miRNA20B (Table 1). MicroRNA involvement is also suggested by analysis of the genes concordantly expressed between DC- and PF-fibroblasts but significantly different in CT. Three microRNAs are putatively implicated: miRNA202, miRNA122, miRNA214 (Table 2).

Validation of the differential expression of select gene products by quantitative RT-PCR

Quantitative real time RT-PCR was performed on the same RNA extracted for the microarray analysis on select genes to validate the microarray results (shown in Figure 5). The genes selected for such confirmation have not previously been reported to be differentially expressed in CT-, PF- or DC-derived fibroblasts. Angiopoietin-like7 mRNA (ANGPTL7) was significantly decreased in DC-derived fibroblasts compared to both PFand CT- derived cells, amongst which no significant difference was seen (Figure 6a). LAMA5 mRNA was similarly least in DC-derived cells, but was also significantly less in PF- compared to DC-derived cells (Figure 6b). In contrast, Shroom 2 message was dramatically increased in DCderived fibroblasts compared to both CT- and PF-derived fibroblasts (Figure 6c). As can be seen by comparing the gene expression patterns in Figures 5 and 6, in each case quantitative RT-PCR findings closely matched the results from microarray analysis.

\section{Discussion}

We previously reported significant differences in gene expression that distinguish fibroblasts obtained from Dupuytren's Contracture cords versus fibroblasts obtained from the normal fascia of patients undergoing surgical therapy for carpal tunnel syndrome [23]. The culture conditions in this study were modified from our previous report through the use of a type- 1 collagen substrate as opposed to an uncoated standard plastic surface. Although the morphological phenotype of the fibroblasts appeared unaffected, many more differentially expressed genes (894 total) were detected in DC versus CT fibroblasts compared to the previous study (40 differentially expressed genes by Illumina BeadArray). Our results can be interpreted to indicate that palmar fascia fibroblasts are sensitive to their culture substrate at a molecular level despite minimal changes in morphological appearance and behavior. These findings can also be interpreted in light of our previous studies indicating that total cellular $§$ catenin levels in DC cells are altered by interactions with a type- 1 collagen substrate [24]. Consistent with this, Ingenuity analysis on

Table 1 Ingenuity analysis of genes concordantly expressed between PF- and CT-derived fibroblasts but significantly different in DC-fibroblasts

\begin{tabular}{|c|c|c|c|c|c|}
\hline ID & Molecules in Network & Score & $\begin{array}{l}\text { Focus } \\
\text { Molecules }\end{array}$ & Top Functions & Canonical Pathways \\
\hline 1 & $\begin{array}{l}\text { ARID4B, ATRX, C1ORF25, CCL7, CDKN1A, CEP350, } \\
\text { EGLN1, EPOR, FAM124B, FBXO9, GRIN1, HDAC1, } \\
\text { HNF1A, IMPA2, KCNE4, MIR106A (includes } \\
\text { EG:406899), MIR17 (includes EG:406952), MIR20B } \\
\text { (includes EG:574032), MXI1, MYC, MYCT1, NFIA, } \\
\text { NFkB (complex), NRG1, PPARA, RORA, SAP130, } \\
\text { SEMA4B, Sin3A, SKP1, SUDS3, TNF, TRIP6, ZNF442, } \\
\text { ZNF33B }\end{array}$ & 53 & 18 & $\begin{array}{l}\text { Cell Death, Cell } \\
\text { Cycle, Cellular } \\
\text { Growth and } \\
\text { Proliferation }\end{array}$ & $\begin{array}{l}\text { DNA Methylation and Transcriptional Repression } \\
\text { Signaling, Toll-like Receptor Signaling, TREM1 } \\
\text { Signaling. Erythropoietin Signaling, PXR/RXR } \\
\text { Activation, LXR/RXR activation, Melatonin Signaling, } \\
\text { Chemokine Signaling, PPAR Signaling, FXR/RXR } \\
\text { Activation, HIF1 } \propto \text { Signaling, Hepatic Cholestasis, Aryl } \\
\text { Hydrocarbon Receptor Signaling, Inositol Phosphate } \\
\text { Metabolism, PPAR } / \text { RXR } \propto \text { Activation, Production of } \\
\text { Nitric oxide and Reactive Oxygen Species in } \\
\text { Macrophages, LPS/L-1 Mediated Inhibition of RXR } \\
\text { Function, Axonal Guidance Signaling }\end{array}$ \\
\hline 2 & HSPB3, LSM14B & 3 & 1 & $\begin{array}{l}\text { Cellular } \\
\text { Compromise, } \\
\text { Cellular Function } \\
\text { and Maintenance }\end{array}$ & \\
\hline
\end{tabular}


Table 2 Ingenuity analysis of genes concordantly expressed between DC- and PF-fibroblasts but significantly different in $\mathrm{CT}$

\begin{tabular}{|c|c|c|c|c|}
\hline$\overline{I D}$ & Molecules & Score & $\begin{array}{l}\text { Focus } \\
\text { Molecules }\end{array}$ & Top Functions \\
\hline 1 & $\begin{array}{l}\text { ABCG5, Ap1, BIRC3, CASP1, Caspase, CCR6, } \\
\text { Creb, CSF2RA (includes EG:1438), DAPP1, } \\
\text { DDOST, DMC1, ERK1/2, HNRNPU, IL1, IL18, } \\
\text { IL12 (complex), IL17RD, Jnk. LEP MECOM, }\end{array}$ & 47 & 25 & $\begin{array}{l}\text { Cell Cycle, DNA Replication, } \\
\text { Recombination, and Repair, } \\
\text { Metabolic Disease }\end{array}$ \\
\hline
\end{tabular}
IL12 (complex), IL17RD, Jnk, LEP, MECOM, NAPB, NFkB (complex), PDGF BB, PNPT1, RAD51, RNF31, SLC12A4, SSTR2, STAT5a/b, SUMO1, TNFSF14, TNFSF15, TP53BP2,

UNC5B, XRCC2

2 ACTR3, ACVR2A, ADCY, AHR, Akt, CAPZA1, CHRNA5, CK2, CTDP1, ERK, FLNC, FSH, ganglioside GD1b, GATA6, hCG, Histone h3, Histone h4, HSD17B7, Lh, MAGT1, Mapk, MIR124, NELF, NME2, P38 MAPK, PI3K, Pka, PPIB, PROS1, RNA polymerase II, SNAPC4, STAR, STMN1, UPP1, USP49 FBXL12, FCAR, FKBP11, GADD45B, IL9, IL24, II12 receptor, ITIH3, ITIH5, ITPA, KIAA0101, MAN2B2 (includes EG:23324), MARK3, MCART1, NFYB, PCNA, PFN2, PGRMC1, PILRA, POLD1, PTGS1, RORA, RRAD, STAT4, TBX21, TERT, TGFB1, TGFBR2, XBP1, ZNF394
DNA Replication, Recombination, and Repair, Drug Metabolism, Endocrine System Development and Function

Cellular Development, Cell Morphology, Cell-Mediated Immune Response

\section{Canonical Pathways}

Pyrimidine Metabolism, Artherosclerosis Signaling, TREM1 Signaling, Glycosphingolipid Biosynthesis-Lactoseries, Death Receptor Signaling, Nucleotide Sugars Metabolism, Aminosugars Metabolism, LXR/RXR Activation, Axonal Guidance Signaling, FXR/RXR Activation, Amyotrophic Lateral Sclerosis Signaling, Endothelin-1 Signaling, N-Glycan Degradation, Cholecystokinin/Gastrinmediated Signaling, p38 MAPK Signaling, Glycosaminoglycan Degradation, T Helper Cell Differentiation, Graft-versus-Host Disease Signaling, Ovarian Cancer Signaling, Chondroitin Sulfate Biosynthesis, Role of Cytokines in Mediating Communication between Immune Cells, Lymphotoxin $\beta$ Receptor Signaling, Role of BRCA1 in DNA Damage Response, Dendritic Cell Maturation, Induction of Apoptosis by HIV1, IL-10 Signaling, JAK/STAT Signaling, GM-CSF Signaling, Hypoxia Signaling in the Cardiovascular System, Communication between Innate and Adaptive Immune Cells, Antiproliferative Role of Somatostatin Receptor 2, Linoleic Acid Metabolism, Acute Phase Response Signaling, Acute Myeloid Leukemia Signaling, leptin Signaling in Obesity, Altered T Cell and B Cell Signaling in Rheumatoid Arthritis, Role of Pattern Recognition Receptors in Recognition of Bacteria and Viruses, PPAR Signaling, Apoptosis Signaling, IL-6 Signaling, Crosstalk between Dendritic Cells and Natural Killer Cells, p53 Signaling, Chronic Myeloid Leukemia Signaling,

Pyrimidine Metabolism, Purine Metabolism, $\mathrm{N}$-Glycan Biosynthesis, Axonal Guidance Signaling, Androgen and Estrogen Metabolism, Factors Promoting Cardiogenesis in Vertebrates, Coagulation System, RhoA Signaling, Role of NANOG in Mammalian Embryonic Stem Cell Pluripotency, AMPK Signaling, EstrogenDependent Breast Signaling, Activation of IRF by Cytosolic Pattern Recognition Receptors, Wnt/B-catenin Signaling, Caveolar-mediated Endocytosis Signaling, Ephrin Recpetor Signaling, NRF2-mediated Oxidative Stress Response, TGF- $\beta$ Signaling, Protein Ubiquitination Pathway, Thrombin Signaling, Regulation of Actin-based Motility by Rho, Virus Entry via Endocytic Pathways, Fcy Receptor-mediated Phagocytosis in Macrophages and Monocytes

Pyrimidine Metabolism, Purine Metabolism, Cleavage and Polyadenylation of Pre-mRNA Eicosanoid Signaling, Endothelin-1 Signaling, N-Glycan Degradation, Arachidonic Acid Metabolism, Ovarian Cancer Signaling, 
Table 2 Ingenuity analysis of genes concordantly expressed between DC- and PF-fibroblasts but significantly different in CT (Continued)

4 Amino acids, BACE1, BLZF1, C1D, CBR3, CCAR1, CDC6, COL4A3BP, CSNK1G2, EXO1, FEN1, GSTA5, HNF4A, MCM4, MCM5, MCM6, MCM8, MEG3 (includes EG:55384), MLH1, MST1, PMM1, PPARG, PPP1R15B, PRKY, PTGR2, RAB2A, RORC, SAP30BP, SFRS11, SORBS1, STK16, TEAD3, TP53, VDAC2, XPNPEP3

5 C12ORF24, C3ORF34, C4ORF43, CBR3, CDKN2AIPNL, CEBPB, CYP1A1, D-glucose, DAG1, DCAF13, ETFDH, F7, FKTN, HNF1A, HNF4A, IL1B, INS, LRSAM1, MAPK8, NCK1, NFkB (complex), PHF23, POFUT1, PRR3, SESN2, SLC25A32, SP1, SRC, TSG101, UBE2D3 (includes EG:7323), UBE2N, ZNF557, ZNF577

6 ATP, BACE1, C14ORF153, CASP3, CRCP, CTSD, EEF2, EIF2AK3, ERBB2, EREG, ERP29, FUT3, GALNT3, ganglioside GD1a, GHR, GRIPAP1, HIVEP2, HLA-DRA, HRAS, IL24, MSH2, MYO9B, N4BP2, NLRP3, ODZ3, PPP1R15A (includes EG:23645), PRTN3, RFX4, RFXANK, Rsk, SERPINE2, Shc, Sod, SULT1A1, TNF

7 C9ORF80, CDC45L, CTSD, DEFB1, EEF2, Eotaxin, ERAP2, GADD45B, GHR, GRM8, HMGA1, IFNB1, IFNG, IRF9, LILRB3 (includes EG:11025), LIMK1, LMNB2, MSH2, MSH3, MYC, ORC1L, ORC2L, ORC6L, PHC2, PNPT1, RHOB, Rock, ROCK2, RPL7, SEPHS2, SERPING1, STK11, TLE1, TMEM70, UTY
$21 \quad 14$

14

Cycle

Tumor Morphology, Cancer, Cell

Metabolism,
2013 Carbohydrate Metabolism, Molecular Transport, Small Molecule Biochemistry

Cellular Assembly and Organization, Tissue Morphology, Cell Death

Cell Cycle, Gene Expression, Inflammatory Response

Cell Signaling, Molecular Transport, Vitamin and Mineral Metabolism

Cell Death, Cell-mediated Immune Response, Cellular Movement

Cellular Development, Cell Cycle, Cell Death
Pyrimidine Metabolism, Purine Metabolism, Cysteine Metabolism, Glycosphingolipid Biosynthesis-Lactoseries, Sulfur Metabolism, Glycosphingolipid BiosynthesisNeolactoseries, O-Glycan Biosynthesis, Keratan Sulfate Biosynthesis, Chondroitin Sulfate Biosynthesis, BMP Signaling Pathway, NRF2-mediated Oxidative Stress Response, EIF2 Signaling

Pyrimidine Metabolism, Nucleotide Sugars Metabolism, Aminosugars Metabolism, Axonal Guidance Signaling, Cholecystokinin/gastrin-mediated Signaling, Complement System, RhoA Signaling, Glycosaminoglycan Degradation, CCR3 Signaling in Eosinophils, Selenoamino Acid Metabolism, Semaphorin Signaling in Neurons, Acute Phase Response Signaling, Ephrin Receptor Signaling, Chemokine Signaling, Leukocyte Extravasation Signaling, VEGF Signaling, Thrombin Signaling

Primary Immunodeficiency Signaling, Starch and Sucrose Metabolism, Atherosclerosis Signaling, Oxidative Phosphorylation, Eicosanoid Signaling, Cyanoamino Acid Metabolism, Stillbene, Coumanine and Lignin Biosynthesis, Phospholipid Degradation, Endothelin-1 Signaling, p38 MAPK signaling, MIF Regulation of Innate Immunity, CCR3 Signaling in Eosinophils, Arachidonic Acid Metabolism, Glycerophospholipid Metabolism, Linoleic Acid Metabolism

Leukocyte Extravasation Signaling
Creatine Kinase, CSNK1A1L, EEF2, EIF2AK2, FOXO1, GAR1, HSP90AB1, KPNB1, KRI1, LAGE3, NHP2, OSGEP, OTUD5, PCNA, PPM1K, RAF1, RAN, RANGRF, RPLP0 (includes EG:6175), RPS6, SNRPE, SPN, SPRY2, SPRYD5, TACR1, TGFBR2, TNFRSF1B, TRAF3, XPO1, XPO5

10 ALPHA AMYLASE, AMY1A, AMY1B, BMP8B, CD44, CSF1, DAG1, DNAH6, DYNLT1, EPO, FOXO1, FOXO3, FYN, glycogen, GRIN1 HCLS1, IL15, INPPL1, KCNH6, LCK, LMOD3, MIR9-1 (includes EG:407046), NMT1, oleic acid, PLCG2, Pld, PLIN5, RPS4Y2, Shc, SHC1, SHCBP1, TDRD1, VCAM1, VEGF
Starch and Sucrose Metabolism, Factor Promoting Cardiogenesis in Vertebrates, Role of NANOG in Mammalian Embryonic Stem Cell Pluripotency, Role of Osteoblasts, Osteoclasts and Chondrocytes in Rheumatoid Arthritis, Basal Cell Carcinoma Signaling, BMP Signaling 
Table 2 Ingenuity analysis of genes concordantly expressed between DC- and PF-fibroblasts but significantly different in CT (Continued)

\begin{tabular}{|c|c|c|c|c|c|}
\hline 11 & $\begin{array}{l}\text { ASB1, BEGAIN, C1ORF9, C8ORF45, CCDC85B, } \\
\text { CTSD, DEFB103A, Eotaxin, EREG, ganglioside } \\
\text { GD1a, ganglioside GD1b, ganglioside GM1, } \\
\text { ganglioside GM2, ganglioside GM3, } \\
\text { ganglioside GT1, GHR, IL32, KIAA0408, LHX4, } \\
\text { MAFF, MAPK1, MIR202 (includes EG:387198), } \\
\text { NDUFA5, NEUROG3, NRP1, PCBP2, PDE4C, } \\
\text { PSAP, ROBO1, SEMA3D, SEMA3E, Sphk, TNF, } \\
\text { ZFP36, ZNF337 }\end{array}$ & 14 & 12 & $\begin{array}{l}\text { Lipid Metabolism, Small } \\
\text { Molecule Biochemistry, Cell } \\
\text { Morphology }\end{array}$ & $\begin{array}{l}\text { Purine Metabolism, Aminosugars } \\
\text { Metabolism, Oxidative Phosphorylation, } \\
\text { Lipid Antigen Presentation by CD1, Axonal } \\
\text { Guidance Signaling, Ubiquinone } \\
\text { Biosynthesis }\end{array}$ \\
\hline 12 & $\begin{array}{l}\text { ACTN1, BCAS2, C16ORF72, COPS2, CTTNBP2, } \\
\text { CTTNBP2NL, DNTTIP2, EGR1, ESR1, ESR2, } \\
\text { FAM40A, FAM40B, FAM63B, G alphai, JUN, } \\
\text { MCC, MCM2, MIR122, MIR214 (includes } \\
\text { EG:406996), MOBKL3, NROB1, NR2F1, NRIP1, } \\
\text { PPP2R1A, PTEN, retinoic acid, RNF122, RP6- } \\
\text { 213H19.1, SIKE1, STK24, STK25, STRN, STRN3, } \\
\text { TRAF3IP3 }\end{array}$ & 9 & 7 & $\begin{array}{l}\text { Gene Expression, Cellular } \\
\text { Development, Cellular Growth } \\
\text { and Proliferation }\end{array}$ & Oxidative Phosphorylation \\
\hline
\end{tabular}

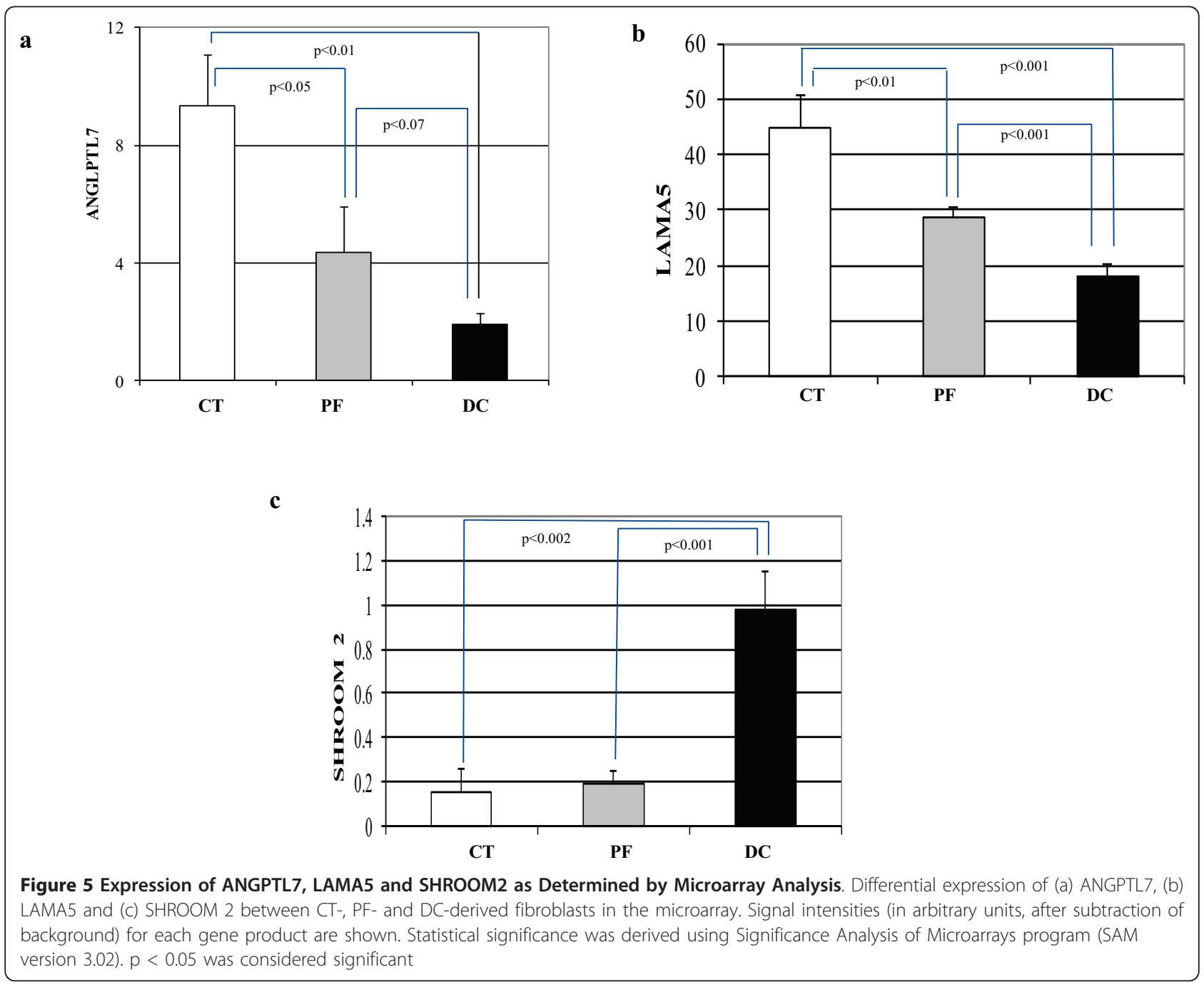




$$
\text { a }
$$

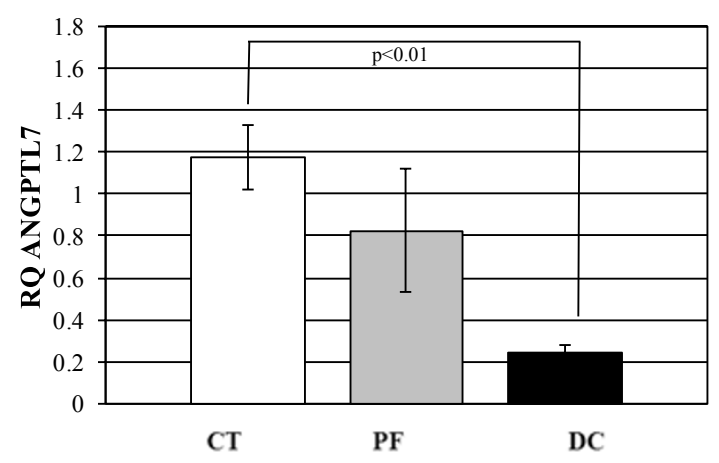

C b

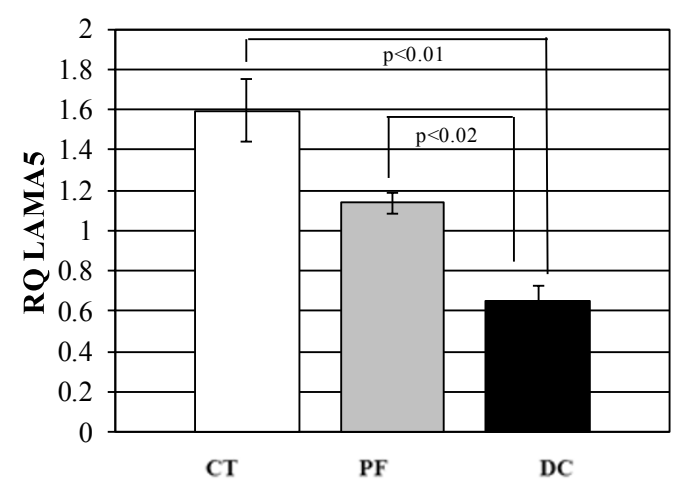

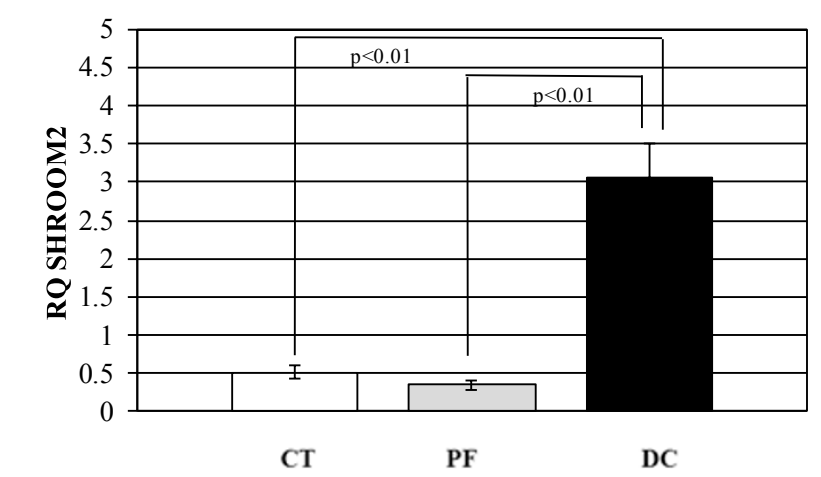

Figure 6 Real time RT-PCR Quantitation of ANGPTL7, LAMA5 and SHROOM2 in CT-, PF-, and DC-derived cells. Differential expression of (a) ANGPTL7, (b) LAMA5 and (c) SHROOM2 was directly confirmed by qRT-PCR in CT-, PF- and DC-derived fibroblasts. Values are mean \pm SEM of two independent experiments performed in triplicate. GAPDH was used as an internal control. Statistical analyses were performed by Student's $t$ test. Relative quantification of gene expression was calculated by comparing $\delta$ Ct values between CT-, PF- and DC- derived fibroblasts. $p<0.05$ was considered significant

genes concordantly expressed between DC- and PF- but significantly different in CT-derived cells suggests the involvement of molecules in the $\mathrm{Wnt} / \beta$-catenin signaling pathway. The gene encoding $\beta$ catenin, CTNNB1, was not amongst the dysregulated genes identified in this analysis, consistent with the primary regulation of cellular $\$$ catenin levels through post-translational mechanisms rather than transcriptional induction [27]. Viewed in combination, our findings suggest that the sensitivity of fibroblasts derived from the palmar fascia of DC patients to their immediate environment is evident at both transcriptomic and proteomic levels. These findings may have profound implications for in vitro culture models designed to replicate DC in vivo and highlight the potentially important effects of culture substrates on cellular phenotypes.

There are at least two non-exclusive, potential explanations for the marked differences in gene expression between primary palmar fascia fibroblasts grown on plastic tissue culture trays and those grown on a type- 1 collagen substrate. The collagen concentration used for these analyses was $1.9 \mathrm{mg} / \mathrm{ml}$, yielding a relatively soft substrate that can be readily contracted by differentiated myofibroblasts in fibroblast populated collagen lattice assays [24]. A stiff and non-deformable substrate, such as tissue culture plastic, may promote myofibroblast differentiation in a similar manner to that induced by increased tissue density [28]. This differentiation may be due, at least in part, to the activation of latent TGF- $\$ 1$ secreted by these cells into their extra-cellular matrix [29]. Under these culture conditions, the differences in gene expression in primary fibroblasts from different tissues may be masked by the changes in gene expression induced during myofibroblast differentiation. In contrast, a soft and readily deformable type-1 collagen substrate may have prevented substrate induced myofibroblast differentiation and allowed for more sensitive detection of the original changes in gene expression that more closely reflect the tissues of origin. Additionally, collagens have been reported to induce a "proliferation permissive" signal through a beta1 integrinmediated PI3 Kinase/Akt pathway in fibroblasts in other 
systems [30]. Interactions with type-1 collagen may, therefore, activate gene transcription through this, and possibly other, pathways in CT, PF and DC cells and induce the differential expression of genes that are not activated in the absence of collagen. Thus, culturing primary fibroblasts on a type- 1 collagen substrate could induce any or all of these effects and explain the marked increase in differentially expressed genes identified in our analyses.

It is notable that each different cell group (DC vs. PF vs. CT) displays a remarkable internal consistency. Six unrelated individuals are compared in each cell group and were found to have highly similar transcriptomic profiles, as evidenced by both hierarchical clustering and concordance mapping. This lends confidence to interpretation of our data as a meaningful representation of the actual differences between the tissue and disease states examined. The most compelling observation is that palmar fascia fibroblasts from phenotypically normal tissue in DC patients closely resemble (but, importantly, are not transcriptomically identical to) cells derived from clearly phenotypically abnormal tissue. As a corollary, both fibroblasts from diseased DC cords and uninvolved DC fascia are markedly transcriptomically dissimilar to fibroblasts from carpal tunnel fascia. This observation is strongly suggestive that a major contributor to the tendency for DC recurrence may be a genetic predisposition towards disease of phenotypically normal palmar fascia in DC patients. Such an interpretation would be consistent with previous reports that DC has a genetic basis $\{$ eg. Hu et al., 2005 [3]\}. Our findings may also reflect on recent reports suggesting that DC cells may be derived from mesenchymal stem cells (MSCs) in perinodular fat and skin [31]. While our analyses do not rule out the possibility that MSCs from these tissues contribute to (or perhaps initiate) disease progression or recurrence, the marked similarity between the transcriptomes of DC and PF cells and their relative dissimilarity to $\mathrm{CT}$ cells strongly implies that the vast majority of cells in DC cord tissue are likely to be derived from PF cells.

These observations make it possible to consider the progression and recurrence of DC in a model based on principles first elucidated in tumor biology, the so-called "two-hit hypothesis" [32] first described by Nordling in 1953 [33]. In such a model we hypothesize that an inherited allelic defect (or possibly a group of allelic defects) constituting a first "hit" alters palmar fascial physiology in DC patients, but not sufficiently so as to lead to the DC nodule/cord phenotype. Progression to frank and active disease would require a second somatic mutation (the second "hit") in the remaining allele in the affected tissue type. Such a model would account for the observation that DC is a heritable disorder, usually presenting later in life (with correspondingly greater chances of having accrued the second requisite mutation), but with widely varying penetrance. Given that both PF and DC cells display hundreds of genes differentially expressed at the mRNA level compared to control CT cells, the putative etiological locus may be involved in transcriptional regulation. Of course, it remains possible that DC is a heterogeneous disorder with a similar phenotype arising from multiple disparate underlying genetic loci, nor does such a model eliminate the possibility of de novo sporadic cases of DC.

These findings also have potential implications for in vitro studies designed to dissect the molecular mechanisms of DC. If patients with a genetic predisposition to develop DC have inherited PF cells that exhibit profound differences in gene expression to normal palmar fascia (CT) cells, then PF cells represent the most clinically relevant controls for testing treatments designed to prevent $\mathrm{DC}$ progression. In contrast, normal palmar fascia cells, such as CT cells, are valuable for analyses designed to identify the molecular characteristics that distinguish DC cells from normal cells. Thus, while identifying differences in signaling pathway activation between DC and CT cells [34] are useful analyses to characterize differences from normality, it is clearly essential that such pathways are also assessed in PF cells before they are considered potential therapeutic targets. A study by Pavelic et al., [35] employing a proteomic approach has identified several proteins interacting in various signaling pathways in DC tissues when compared to unaffected palmar fascia from DC patients. This identification of protein-protein interaction potentially opens new therapeutic targets to reduce the recurrence of DC. Optimally, comparisons of DC cells to both CT and PF cells are likely to provide different, but equally valuable, insights into DC progression and recurrence that will allow researchers to distinguish and identify molecular or other therapies that target DC, but not PF or CT, cells.

The remarkable internal consistency between samples in the microarray was also reflected in the findings obtained from real time RT-PCR assays used to validate the microarray results. In seeking potentially novel targets to limit the progression of DC, we chose to directly examine three select genes that have not been previously implicated in the progression or recurrence of DC. Human ANGPTL7 had been characterized as a potent target gene of the $\mathrm{WNT} / \beta$-catenin signaling pathway and currently is a pharmacogenomics target in the fields of oncology and regenerative medicine [36]. Studies by Kuchtey et al., (2008) [37] and subsequent studies by Comes et al., (2010) [38] showed that ANGPTL7 has the potential to alter extracellular matrix formation, a finding that may also be relevant to DC. We find that in DC-derived fibroblasts ANGPTL7 mRNA is substantially decreased in comparison to CT- and PF-derived fibroblasts (Figure 5a and 6a). The functional importance of 
ANGPTL7 in the biology of DC is unknown and further studies are required to understand its role in DC progression.

We also directly examined the extracellular matrix protein laminin isoform, laminin alpha5 (LAMA5). Laminin isoforms have been shown to play crucial roles in modulating cell adhesion, proliferation, differentiation, and migration in normal and pathological states by interacting with other extracellular matrix components $[39,40]$. In both microarray and by quantitative RT-PCR we found that LAMA5 mRNA expression was specifically increased in CT- and PF-derived cells compared to DCderived fibroblasts (Figure $5 \mathrm{~b}$ and $6 \mathrm{~b}$ ). A previous study by Kosmehl et al., (1995) [41] showed higher expression of laminin A, as well as M, B1, B2 and S chains, in Dupuytren nodular tissues, but LAMA5 expression in cord tissue is unknown. Further dissection of the functional significance LAMA5 might provide valuable insights for manipulation to limit the progression of DC.

In contrast to ANGPTL7 and LAMA5, direct examination of Shroom 2 message showed a marked increase in DC-derived cells ( 5-6-fold) compared to CT- or PFderived fibroblasts, again mirroring the findings from the microarray data (Figures 5c \&6c). Shroom family proteins are reported to interact with actin and actin polymerization is required for their function $[42,43]$. Moreover, members of the Shroom family (mainly Shrooms 1, 2 and 3) cause accumulation of $\gamma$-tubulin, a microtubule nucleating protein, at the apical surface of epithelial cells [44]. Based on these and other findings it has been suggested that Shroom genes function in the regulated control of cytoskeletal molecules and thereby aid in cell morphogenesis. We have found that interference with actin pathways can affect Dupuytren's fibroblast contractility (Satish et al., manuscript in preparation) and enhanced levels of Shroom 2 may, be one of the factors that alter actin-mediated cell contraction in DC, potentially identifying it as an attractive target through which to inhibit disease progression or recurrence.

Ingenuity analysis of our differentially expressed gene sets has identified multiple microRNAs that may play a role in DC pathophysiology, although direct experimental evidence is as yet lacking. Recently Mosakhani et al., [45] for the first time examined expression of microRNAs in affected DC tissues, comparing them to normal fibroblasts in culture and normal fascia obtained from hand trauma and carpal tunnel patients. Curiously, although they identified dozens of differentially expressed microRNAs, none of the microRNAs suggested by our Ingenuity analysis appeared in their screen. This may be due to the disparate natures of the sample sources (ie. tissues containing a mixture of cells of mesenchymal, endothelial and other lineages versus primary fibroblasts in culture) or other reasons, such as the absence of mechanical stress to activate mechanoreceptors, or additional extra-cellular matrix components and matricellular molecules that may, directly or indirectly, regulate microRNA transcription. We anticipate that additional analyses incorporating some or all of these potential effectors of DC development in vivo will be required to establish the full relevance of the differential gene expression patterns we report here.

\section{Conclusions}

These data show that the transcriptomic profiles of DCdisease fibroblasts and fibroblasts from unaffected palmar fascia in DC patients are highly similar, and differ significantly from the transcriptomic profiles of fibroblasts from the palmar fascia of patients undergoing carpal tunnel release. These observations are further evidence of an inherent molecular pathophysiology in DC disease fibroblasts that may help to explain the progression of the disease and its tendency to recurrence.

\section{Additional material}

Additional file 1: Differences in individual gene expression levels identified through SAM analysis.

\section{Acknowledgements}

The authors thank Allegheny-Singer Research Institute and Pennsylvania Department of Health for their financial support towards this study. We would also like to extend our sincere thanks to Dr. Yan Wu for her excellent technical assistance in procuring and maintaining the cell lines.

\section{Author details}

'Department of Surgery, Division of Plastic Surgery, University of Pittsburgh Medical Center, Pittsburgh, PA, USA. '2Department of Pathology, University of Pittsburgh, Pittsburgh, PA, USA. ${ }^{3}$ Center for Genomic Sciences, AlleghenySinger Research Institute, Allegheny General Hospital, Pittsburgh, PA, USA. ${ }^{4}$ Cell and Molecular Biology Laboratory of the Hand and Upper Limb Centre, St. Joseph's Hospital, London, ON, Canada. ${ }^{5}$ Division of Upper Extremity Surgery, Department of Orthopaedics, Allegheny General Hospital, Pittsburgh, PA, USA.

\section{Authors' contributions}

LS, SK, DO'G and BSG conceived and designed the experiments. LV, AN, CR, LS and SJ performed the experiments. PHG performed the real time RT-PCR experiments. Analysis tools were contributed by WAL and JMKB. Data analyses were performed by WAL, JMKB, LS, SK, DO'G, BSG. Cell cultures/ materials were contributed by DO'G, BSG, MEB, GDE. The manuscript was drafted by SK, LS and WAL. The manuscript was critically reviewed by MEB, $L V, A N, C R, D O \prime G, B S G, G D E$. All authors have read and approved the final manuscript.

\section{Competing interests}

The authors declare that they have no competing interests.

Received: 6 July 2011 Accepted: 4 May 2012 Published: 4 May 2012

\section{References}

1. Chiu HF, McFarlane RM: Pathogenesis of Dupuytren's contracture a correlative clinical-pathological study. J Hand Surg [Am] 1978, 3:1-10

2. Ross DC: Epidemiology of Dupuytren's disease. Hand Clinics 1999, 15:53-62. 
3. Hu FZ, Nystrom A, Ahmed A, Palmquist M, Dopico R, Mossberg I, Gladitz J Rayner M, Post JC, Ehrlich GD, Preston RA: Mapping of an autosomal dominant gene for Dupuytren's contracture to chromosome $16 \mathrm{q}$ in a Swedish family. Clin Genet 2005, 68:424-429.

4. Hindocha S, John S, Stanley JK, Watson SJ, Bayat A: The heritability of Dupuytren's disease: familial aggregation and its clinical significance. $J$ Hand Surg [Am] 2006, 31:204-210.

5. Geoghegan JM, Forbes J, Clark DI, Smith C, Hubbard R: Dupuytren's disease risk factors. J Hand Surg [Br] 2004, 29:423-426.

6. Godtfredsen NS, Lucht H, Prescott E, Sorensen TI, Gronbaek M: A prospective study linked both alcohol and tobacco to Dupuytren's disease. J Clin Epidemiol 2004, 57:858-863.

7. Bergenudd H, Lindgarde F, Nilsson BE: Prevalence of Dupuytren's contracture and its correlation with degenerative changes of the hands and feet and with criteria of general health. J Hand Surg [Br] 1993, 18:254-257.

8. Bennett B: Dupuytren's contracture in manual workers. Br J Ind Med 1982, 39:98-100.

9. Frey M: Risks and prevention of Dupuytren's contracture. Lancet 1997, 350:1568.

10. Kloen P: New insights in the development of Dupuytren's Contracture: A review. Br J Plast Surg 1999, 52:629-635.

11. Akyol A, Kiylioglu N, Copcu E, Guney E, Aydeniz A: Is diabetes mellitus type 2 a risk factor for Dupuytren's contracture in the Mediterranean region? Plast Reconstr Surg 2006, 117:2105-2106.

12. Ketchum LD, Donahue TK: The injection of nodules of Dupuytren's disease with triamcinolone acetonide. J Hand Surg 2000, , 25A:: 1157-1162.

13. Pittet $B$, Rubbia-Brandt $L$, Desmoulière $A$, Sappino $A P$, Roggero $P$, Guerret $S$, Grimaud JA, Lacher R, Montandon D, Gabbiani G: Effect of gammainterferon on the clinical and biologic evolution of hypertrophic scars and Dupuytren's disease: an open pilot study. Plast Reconstr Surg 1994, 93:1224-1235

14. Richard H: Dupuytren's contracture treated with vitamin E. Br Med J 1952, 1:1320-1321.

15. Vuopala U, Kaipainen W: DMSO in the treatment of Dupuytren's contracture. A therapeutic experiment. Acta Rheum Scand 1971, 17:61-62.

16. Stiles P: Ultrasonic therapy in Dupuytren's contracture. J Bone Joint Surg Br 1966, 48:452-454.

17. Hurst LC, Badalamente MA: Nonoperative treatment of Dupuytren's disease. Hand Clinics 1999, 15:97-107.

18. Badalamente MA, Hurst LC: Efficacy and safety of injectable mixed collagenase subtypes in the treatment of Dupuytren's contracture. J Hand Surg [Am] 2007, 32:767-774.

19. Hurst LC, Badalamente MA, Hentz VR, Hotchkiss RN, Kaplan FT, Meals RA, Smith TM, Rodzvilla J, CORD I Study Group: Injectable collagenase clostridium for Dupuytren's contracture. N Engl J Med 2009, 361:968-979.

20. Roush TF, Stern PJ: Results following surgery for recurrent Dupuytren's disease. J Hand Surg [Am] 2000, 25:291-296.

21. Au-Yong IT, Wildin CJ, Dias JJ, Page RE: A review of common practice in Dupuytren surgery. Tech Hand Up Extrem Surg 2005, 9:178-187.

22. Howard JC, Varallo VM, Ross DC, Faber KJ, Roth JH, Seney S, Gan BS: Wound healing-associated proteins $\mathrm{Hsp} 47$ and fibronectin are elevated in Dupuytren's contracture. J Surg Res 2004, 117:232-238.

23. Satish L, LaFramboise WA, O'Gorman DB, Johnson S, Janto B, Gan BS, Baratz ME, Hu FZ, Post JC, Ehrlich GD, Kathju S: Identification of differentially expressed genes in fibroblasts derived from patients with Dupuytrens Contracture. BMC Med Genomics 2008, 1:10.

24. Vi L, Njarlangattil A, Wu Y, Gan BS, O'Gorman DB: Type-1 collagen differentially alters beta-catenin accumulation in primary Dupuytren's disease cord and adjacent palmar fascia cells. BMC Musculoskelet Disord 2009, 10:72.

25. Tusher VG, Tibshirani R, Chu G: Significance analysis of microarrays applied to the ionizing radiation response. Proc Natl Acad Sci USA 2001, 98:5116-5121[http://www-stat.Stanford.edu/ tibs/SAM/]

26. Sneath PHA, Sokal RR: Numerical Taxonomy San Francisco: W.H. Free-man; 1973.

27. Bowley E, O'Gorman DB, Gan BS: $\beta$-catenin Signaling in Fibroproliferative Disease. Journal of Surgical Research 2007, 138(1):141-150.
28. Hinz B: Tissue stiffness, latent TGF-beta1 activation, and mechanical signal transduction: implications for the pathogenesis and treatment of fibrosis. Curr Rheumatol Rep 2009, 11:120-126.

29. Wipff PJ, Rifkin DB, Meister JJ, Hinz B: Myofibroblast contraction activates latent TGF-beta1 from the extracellular matrix. J Cell Biol 2007, 179:1311-1323.

30. Nho RS, Kahm J: beta1-Integrin-collagen interaction suppresses FoxO3a by the corordination of Akt and PP2A. J Biol Chem 2010, 285:14195-209.

31. Iqbal SA, Manning C, Syed F, Kolluru V, Hayton M, Watson S, Bayat A: Identification of Mesenchymal Stem Cells in Perinodular Fat and Skin in Dupuytren's Disease: A Potential Source of Myofibroblasts with Implications for Pathogenesis and Therapy. Stem Cells Dev 2012, 21(4):609-622.

32. Knudson AG Jr: Mutation and cancer: statistical study of retinoblastoma. Proc Natl Acad Sci USA 1971, 68:820-823.

33. Nordling CO: A new theory on cancer-inducing mechanism. $\mathrm{Br} J$ Cancer 1953, 7:68-72.

34. Krause $C$, Kloen $P$, Ten Dijke $P$ : Elevated transforming growth factor $\beta$ and mitogen-activated protein kinase pathways mediate fibrotic traits of Dupuytren's disease fibroblasts. Fibrogenesis Tissue Repair 2011, 4(1):14.

35. Kraljevic Pavelic S, Sedic M, Hock K, Vucinic S, Jurisic D, Gehrig P, Scott M, Schlapbach R, Cacev T, Kapitanovic S, Pavelic K: An integrated proteomics approach for studying the molecular pathogenesis of Dupuytren's disease. J Pathol 2009, 217(4):524-33.

36. Katoh $Y$, Katoh $\mathrm{M}$ : Comparative integromics on angiopoietin family members. Int J Mol Med 2006, 17:1145-1149.

37. Kuchtey J, Källberg ME, Gelatt KN, Rinkoski T, Komàromy AM, Kuchtey RW: Angiopoietin-like 7 secretion is induced by glaucoma stimuli and its concentration is elevated in glaucomatous aqueous humor. Invest Opthalmol Vis Sci 2008, 49:3438-3448.

38. Comes N, Buie LK, Borràs T: Evidence for a role of ANGPTL7 in extracellular matrix formation of the human trabecular meshwork: implications for glaucoma. Genes to Cells 2011, 16:243-259.

39. Scheele $S$, Nystrom A, Durbeej $M$, et al: Laminin isoforms in development and disease. J Mol Med 2007, 85:825-836.

40. Tzu J, Marinkovich MP: Bridging structure with function: structural, regulatory, and developmental role of laminins. Int J Biochem Cell Biol 2008, 40:199-214.

41. Kosmehl H, Berndt A, Katenkamp D, Mandel U, Bohle R, Gabler U, Celeda D: Differential expression of fibronectin splice variants, oncofetal glycosylated fibronectin and laminin isoforms in nodular palmar fibromatosis. Pathol Res Pract 1995, 191:1105-1113.

42. Dietz ML, Bernaciak TM, Vendetti F, Kielec JM, Hildebrand JD: Differential actin-dependent localization modulates the evolutionarily conserved activity of Shroom family proteins. J Biol Chem 2006, 281:20542-20554.

43. Yoder M, Hildebrand JD: Shroom4 (Kiaa 1202) is an actin-associated protein implicated in cytokeletal organization. Cell Motil Cytoskeleton 2007, 64:49-63.

44. Lee C, Scherr HM, Wallingford JB: Shroom family proteins regulate gamma-tubulin distribution and microtubule architecture during epithelial cell shape change. Development 2007, 134:1431-1441.

45. Mosakhani N, Guled M, Lahti L, Borze I, Forsman M, Pääkkönen V, Ryhänen J, Knuutila S: Unique microRNA profile in Dupuytren's contracture supports deregulation of $\beta$-catenin pathway. Modern Pathology 2010, 23:1544-1552.

\section{Pre-publication history}

The pre-publication history for this paper can be accessed here: http://www.biomedcentral.com/1755-8794/5/15/prepub

\section{doi:10.1186/1755-8794-5-15}

Cite this article as: Satish et al:: Fibroblasts from phenotypically normal palmar fascia exhibit molecular profiles highly similar to fibroblasts from active disease in Dupuytren's Contracture. BMC Medical Genomics 2012 5:15. 